DAMTP-93-48

EFI-93-54

hep-th/9309146

21 September, 1993

\title{
Null vectors, 3-point and 4-point functions in conformal field theory ${ }^{1}$
}

\author{
P. Bowcock \\ Enrico Fermi Institute, University of Chicago, Chicago, IL 60637, U.S.A. ${ }^{2}$ \\ and \\ G. M. T. Watts \\ St. John's College, St. John's Street, Cambridge, CB2 1TP, U. K. \\ and \\ DAMTP, University of Cambridge, Silver Street, Cambridge, CB3 9EW, U. K.
}

\begin{abstract}
We consider 3-point and 4-point correlation functions in a conformal field theory with a W-algebra symmetry. Whereas in a theory with only Virasoro symmetry the three point functions of descendants fields are uniquely determined by the three point function of the corresponding primary fields this is not the case for a theory with $W_{3}$ algebra symmetry. The generic 3-point functions of $\mathrm{W}$-descendant fields have a countable degree of arbitrariness. We find, however, that if one of the fields belongs to a representation with null states that this has implications for the 3-point functions. In particular if one of the representations is doubly-degenerate then the 3-point function is determined up to an overall constant. We extend our analysis to 4-point functions and find that if two of the W-primary fields are doubly degenerate then the intermediate channels are limited to a finite set and that the corresponding chiral blocks are determined up to an overall constant. This corresponds to the existence of a linear differential equation for the chiral blocks with two completely degenerate fields as has been found in the work of Bajnok et al.
\end{abstract}

In this talk I would like to discuss some features of correlation functions in two-dimensional conformal field theory with extended conformal symmetry algebras, and show that there are some new and interesting results in this area. In the first half I shall review the theory in the case where the chiral algebra is the Virasoro algebra and subsequently discuss the $W_{3}$ algebra. This analysis was inspired by the results on $W$-algebra correlation functions of Bajnok et al. [1]

\footnotetext{
${ }^{1}$ Talk presented by GMTW at the III International conference on Mathematical Physics, String Theory and Quantum Gravity, Alushta, Ukraine

${ }^{2}$ Address from 1 Oct: Dept. Math. Sci. Univ. Durham, South Road, Durham, DH1 3LE, U. K.
} 


\subsection{VIRASORO ALGEBRA REPRESENTATION THEORY}

The Virasoro algebra has generators $L_{m}$ with commutation relations

$$
\left[L_{m}, L_{n}\right]=\frac{c}{12} m\left(m^{2}-1\right) \delta_{m+n, 0}+(m-n) L_{m+n} .
$$

There are two sorts of representations of interest.

Highest weight representations have the spectrum of $L_{0}$ bounded from below. The Verma module $M_{h, c}$ is a highest weight representation and is spanned by the states

$$
L_{i_{1}} \ldots L_{i_{k}}|h\rangle, \quad i_{j} \leq i_{j+1}<0
$$

where $|h\rangle$ satisfies

$$
L_{m}|h\rangle=h \delta_{m, 0}|h\rangle, \quad m \geq 0 .
$$

For generic $h, c, M_{h, c}$ is irreducible. If however $h$ and $c$ satisfy

$$
c=c(t)=13-6 t-6 / t, \quad h=h_{r, s}(t)=\frac{(r t-s)^{2}-(t-1)^{2}}{4 t},
$$

for some values of $t \in \mathbb{R}, r, s \in \mathbb{N}$, then $M_{h_{r, s}(t), c(t)}$ is reducible with an invariant submodule generated by a highest weight state $N_{r, s}\left|h_{r, s}\right\rangle$ at level $r s$ (see ref. [2]). The Virasoro minimal models have $c$ given by eq. (1.4) with $t=\sqrt{p / q}, p, q \in \mathbb{N}, p, q$ coprime, and the fields in the models have $h=h_{r, s}$ with $r, s \in \mathbb{Z}, 0<r<q, 0<s<p$ (see [3]).

If $M_{h, c}$ is reducible than the irreducible highest weight representation $L_{h, c}$ is the quotient of $M_{h, c}$ by its maximal invariant submodule, which is the union of Verma modules generated by embedded highest weight states [4]. For physical reasons one expects the Hilbert space of a theory to be composed of irreducible representations and that the null vectors decouple from all correlation functions.

There are also field representations, of which the simplest is a primary field ${ }^{1}$. A primary field $\phi_{h}^{(y)}$ is labelled by $h$ and $y$ and satisfies

$$
\begin{gathered}
{\left[L_{m}, \phi_{h}^{(y)}(z)\right]=z^{m+1} \partial \phi_{h}^{(y)}(z)+h(m+1) z^{m} \phi_{h}^{(y)}(z)} \\
\phi_{h}^{(y)}(z)=\sum_{n \in \mathbb{Z}} z^{y-n} \phi(h, y)_{n}
\end{gathered}
$$

although we shall often drop the dependence on $y$ when it is clear from context. Heuristically a field $\Phi(z)$ satisfies

$$
\Phi(z)|0\rangle=e^{z L_{-1}}|\Phi\rangle
$$

where $|\Phi\rangle$ is a state in a highest weight representation. A primary field $\phi_{h}$ corresponds to $|\Phi\rangle=|h\rangle$ being a highest weight state.

\subsection{3 -POINT FUNCTIONS}

In ref. [6] Feigin and Fuchs showed us how to consider three-point functions as a map from a Verma module $M_{h, c}$ to $\mathbb{C}$,

$$
|\psi\rangle \mapsto\left\langle h_{2}\left|\phi_{h_{3}}^{(y)}(z)\right| \psi\right\rangle, \quad y=\left(h_{2}-h_{3}-h_{1}\right), \quad \psi \in M_{h, c}
$$

\footnotetext{
${ }^{1}$ This representation is the same as the $F_{\lambda, \mu}$ in [5]
} 


$$
\left\langle h_{2}\left|\phi_{h_{3}}(z)\right| h\right\rangle=C z^{h_{2}-h_{3}-h_{1}}
$$

where $C$ is an arbitrary constant and $z$ a non-zero complex number. Following ref. [6], we can easily evaluate (1.8) by considering the following combinations of generators,

$$
e_{m}(z)=L_{m}-2 z L_{m-1}+z^{2} L_{m-2}, \quad e_{0}^{\prime}(z)=z^{2} L_{-2}-2 z L_{-1}+L_{0}, \quad e_{0}^{\prime \prime}(z)=z^{2} L_{-2}-z L_{-1} .
$$

These have the property that

$$
\left\langle h_{2}\right| \phi_{h_{3}}(z) e_{m}(z)=0, m \leq-1, \quad\left\langle h_{2}\right| \phi_{h_{3}}(z)\left(e_{0}^{\prime}(z)-h_{2}\right)=0, \quad\left\langle h_{2}\right| \phi_{h_{3}}(z)\left(e_{0}^{\prime \prime}(z)-h_{3}\right)=0 .
$$

We shall often consider the case $z=1$ in which case we drop the $z$ dependence in the generators $e_{0}^{\prime}, e_{0}^{\prime \prime}, e_{m}$.

The Verma module $M_{h, c}$ can be spanned by states of the form

$$
\left(e_{0}^{\prime}\right)^{a}\left(e_{0}^{\prime \prime}\right)^{b} e_{i_{1}} \ldots e_{i_{j}}|h\rangle, \quad i_{k} \leq i_{k+1} \leq-1 .
$$

As a consequence, any state $|\psi\rangle$ in $M_{h, c}$ can be written in the form

$$
|\psi\rangle=p\left(e_{0}^{\prime}, e_{0}^{\prime \prime} ; e_{-1}, e_{-2}, \ldots\right)|h\rangle,
$$

where $p$ is a polynomial. The three point functions of this state is very easy to calculate,

$$
\left\langle h_{2}\left|\phi_{h_{3}}(1)\right| \psi\right\rangle=p\left(h_{2}, h_{3} ; 0,0, \ldots\right) C \text {. }
$$

\subsection{3 -POINT FUNCTIONS AND NULL VECTORS}

As stated above, Feigin and Fuchs defined a three point function initially on a Verma module. If this Verma module is reducible, then we must check whether we can restrict this map to the irreducible representation, that is that whether the null vectors decouple. The null vectors can of course also be written in the form (1.12),

$$
N_{p, q}=N_{p, q}\left(e_{0}^{\prime}, e_{0}^{\prime \prime} ; e_{-1}, \ldots\right),
$$

and so from eqn. (1.14)

$$
\left\langle h_{2}\left|\phi_{h_{3}}(1) N_{p, q}\right| h_{p, q}\right\rangle=N_{p, q}\left(h_{2}, h_{3} ; 0,0, \ldots\right) C .
$$

The requirement that the 3-point function can be restricted from the Verma module to the irreducible representation is that the polynomials $N_{p, q}\left(h_{2}, h_{3} ; 0,0, \ldots\right)$ vanish for each highest weight state $N_{p, q}\left|h_{p, q}\right\rangle$ in $M_{h, c}$. If there is a single such highest weight states in $M_{h, c}$ then if $h_{3}$ is given, $h_{2}$ is restricted to a finite set by this requirement. If there is an additional second independent null vector as is the case for the representations arising in the minimal models then this will further restrict the allowed fusions and for those representations which occur in the minimal models the fusions are restricted to a finite set of allowed pairs $\left\{h_{2}, h_{3}\right\}$ (see [6]). The polynomials $N_{p, q}\left(h_{2}, h_{3} ; 0,0, \ldots\right)$ have been found for all $p, q$ indirectly by Feigin and Fuchs in ref. [6], directly for $p=1$ or $q=1$ by Langlands in [7] and by Bauer et al. in [8] and directly by Kent for all $p, q$ in [9]. These polynomials give the usual fusion rules for the Virasoro algebra as predicted in [3]. 


\subsection{4-POINT FUNCTIONS}

A four point function of primary fields is heuristically something of the form

$$
\left\langle h_{2}\left|\phi_{h_{3}}(1) \phi_{h_{4}}(z)\right| h_{1}\right\rangle
$$

A chiral block is given by projecting onto a single intermediate representation, $L_{h_{5}, c}$. We can of course write this projector using an orthonormal basis $\left|\psi_{\iota}\right\rangle$ for $L_{h_{5}, c}$,

$$
\pi=\sum_{\iota}\left|\psi_{\iota}\right\rangle\left\langle\psi_{\iota}\right|
$$

The chiral block $B\left(h_{2}, h_{3}, h_{4}, h_{1} ; h_{5}\right)$ is then given as

$$
B\left(h_{2}, h_{3}, h_{4}, h_{1} ; h_{5}\right)=\sum_{\iota}\left\langle h_{2}\left|\phi_{h_{3}}(1)\right| \psi_{\iota}\right\rangle\left\langle\psi_{\iota}\left|\phi_{h_{4}}(z)\right| h_{1}\right\rangle
$$

Since the 3-point functions in this chiral block are determined up to an overall constant, we find that the chiral block is itself determined by $\left\{h_{i}, c\right\}$ up to an overall scale. Let us consider the consequences if one of the Verma modules $M_{h_{i}, c}, i=1,2,3,4$, is reducible. Without loss of generality, let $h_{1}=h_{p, q}(t), c=c(t)$ so that there is a null state in $M_{h_{1}, c}$, which must decouple from the correlation function. This means that the 3 -point function

$$
\left\langle h_{5}\left|\phi_{h_{4}}(z) N_{p, q}\right| h_{p, q}\right\rangle
$$

must vanish and so we obtain the restriction that $h_{5}$ lies in a finite set. Since the 4-point function is given as a sum over chiral blocks, we see that the 4-point function lies in a finitedimensional space, and can be given as the solution of a linear ordinary differential equation in $z$. This is of course the usual way that the 4-point functions are presented, as solutions of the differential equations expressing the decoupling of the null vector, as in ref. [3]; we present the results this way in order to contrast them with the corresponding results for the $W_{3}$ algebra.

\section{$2.0 \quad W_{3}$ Algebra representation theory}

The $W_{3}$ algebra was introduced by Zamolodchikov in ref. [10] and is the simplest of the extended algebras which still displays interesting properties. It has generators $L_{m}, Q_{m}$ with commutation relations

$$
\begin{aligned}
{\left[L_{m}, Q_{n}\right]=} & (2 m-n) Q_{m+n} \\
{\left[Q_{m}, Q_{n}\right]=} & \frac{(22+5 c)}{48} \frac{c}{3 \cdot 5 !}\left(m^{2}-4\right)\left(m^{2}-1\right) m \delta_{m+n} \\
& +\frac{1}{3}(m-n) \Lambda_{m+n}+\frac{(22+5 c)}{48} \frac{(m-n)}{30}\left(2 m^{2}-m n+2 n^{2}-8\right) L_{m+n}
\end{aligned}
$$

where

$$
\Lambda_{m}=\sum_{p>-2} L_{m-p} L_{p}+\sum_{p \leq-2} L_{p} L_{m-p}-\frac{3}{10}(m+2)(m+3) L_{m},
$$

and $c$ is a central element. The fact that this is not a Lie algebra leads to many interesting problems. 
The representation theory of the $W_{3}$ algebra can be developed in analogy with that of the Virasoro algebra. A $W_{3}$ highest weight vector $|h, q\rangle$ satisfies

$$
L_{m}|h, q\rangle=\delta_{m, 0} h|h, q\rangle, Q_{m}|h, q\rangle=\delta_{m, 0} q|h, q\rangle, m \geq 0 .
$$

The Verma module $M_{h, q, c}$ of the $W_{3}$-algebra is spanned by states of the form

$$
L_{i_{1}} \ldots L_{i_{j}} Q_{k_{1}} \ldots Q_{k_{l}}|h, q\rangle, \quad i_{m} \leq i_{m+1} \leq-1, k_{m} \leq k_{m+1} \leq-1 \text {, }
$$

and by the usual abuse of notation the central element $c$ takes the value $c$. If the Verma module is reducible, then the irreducible representation $L_{h, q, c}$ is the quotient of the Verma module by its maximal invariant submodule (note this submodule does not have to be the union of Verma modules generated by highest weight states, see e.g. ref. [11]).

We can parameterise the weights of a W-highest weight vector as follows [12],

$$
h=\frac{1}{3}\left(x^{2}+x y+y^{2}-3 a^{2}\right), w=\frac{1}{27}(x-y)(2 x+y)(x+2 y),
$$

where we define $a, \alpha_{ \pm}$by

$$
c=2-24 a^{2}, \alpha_{ \pm}^{2}-\alpha_{ \pm} a-1=0 .
$$

The condition that $M_{h, q, c}$ has a null vector with eigenvalues $h^{\prime}, q^{\prime}$ is that we can find some $x, y$ such that $h, q$ are given by eqns. (2.25) and $x$ satisfies

$$
x=r \alpha_{+}+s \alpha_{-}, \quad r, s \in \mathbb{N}, \quad r s>0,
$$

in which case $h^{\prime}, q^{\prime}$ are given by eqns. (2.25) with $x^{\prime}=x-2 r \alpha_{+}, y^{\prime}=y+r \alpha_{+}$. The $W_{3}$ minimal models are those which have $\alpha_{+}=\sqrt{p / q}, p, q \in \mathbb{N}, p, q$ coprime, and the fields in these models have $x=r \alpha_{+}+s \alpha_{-}, y=t \alpha_{+}+u \alpha_{-}, 0<r, s, t, u, r+t<q, s+u<p$ (see [13]). Of these minimal models those with $p=m+1, q=m, m \geq 3$ are unitary since they can be constructed in the explicitly unitary coset construction [14].

The highest weight representation theory of the $W_{3}$ algebra is analogous to that of the Virasoro and the Kac-Moody algebras; primary fields of the $W_{3}$ algebra are not as easy to characterise as those of the Virasoro however.

If we denote the field corresponding to the state $X_{-n}|\Phi\rangle$ by $\hat{X}_{-n} \Phi(z)$, then a $W_{3}$-primary field corresponding to a highest weight state $|h, q\rangle$ should satisfy

$$
\left[Q_{m}, \phi_{h, q}(z)\right]=z^{m}\left\{\frac{1}{2} q(m+1)(m+2)+(m+2) z \hat{Q}_{-1}+z^{2} \hat{Q}_{-2}\right\} \phi_{h, q}(z),
$$

where $\hat{Q}_{-1} \phi, \hat{Q}_{-2} \phi$ are new fields. For generic values of $h, q$ and $c$ it is not possible to find a finite set of fields which closes under the action of the $W_{3}$ algebra. However, we can again use the trick of Feigin and Fuchs and introduce the combinations of generators

$$
\begin{aligned}
f_{m}(z) & =Q_{m}-3 z Q_{m-1}+3 z^{2} Q_{m-2}-z^{3} Q_{m-3}, \quad m \leq-1 \\
f_{0}^{\prime}(z) & =Q_{0}-3 z Q_{-1}+3 z^{2} Q_{-2}-z^{3} Q_{-3} \\
f_{0}^{\prime \prime}(z) & =-z Q_{-1}+2 z^{2} Q_{-2}-z^{3} Q_{-3},
\end{aligned}
$$


which satisfy

$$
\begin{aligned}
& \left\langle h_{2}, q_{2}\right| \phi_{h_{3}, q_{3}}(z) f_{m}(z)=0, \quad m \leq-1 \\
& \left\langle h_{2}, q_{2}\right| \phi_{h_{3}, q_{3}}(z)\left(f_{0}^{\prime}(z)-q_{2}\right)=0 \\
& \left\langle h_{2}, q_{2}\right| \phi_{h_{3}, q_{3}}(z)\left(f_{0}^{\prime \prime}(z)-q_{3}\right)=0 .
\end{aligned}
$$

Again let us put $z=1$ and drop the dependence from the generators $f_{m}$. The problem now with evaluating three point functions is that one cannot span a Verma module using only the combinations $\left\{e_{0}^{\prime}, e_{0}^{\prime \prime}, e_{m} ; q_{0}^{\prime}, q_{0}^{\prime \prime}, q_{m}\right\}$, one also has to consider the mode $Q_{-1}$ separately. A basis of the Verma module is given by the states

$$
\begin{gathered}
e_{i_{1}} \ldots e_{i_{j}} f_{k_{1}} \ldots f_{k_{l}}\left(e_{0}^{\prime}\right)^{a}\left(e_{0}^{\prime \prime}\right)^{b}\left(f_{0}^{\prime}\right)^{c}\left(f_{0}^{\prime \prime}\right)^{d}\left(Q_{-1}\right)^{e}|h, q\rangle, \\
i_{m} \leq i_{m+1} \leq-1, k_{m} \leq k_{m+1} \leq-1
\end{gathered}
$$

The three-point function of the state

$$
|\psi\rangle=\sum_{a=0} p_{a}\left(e_{0}^{\prime}, e_{0}^{\prime \prime}, e_{-1}, \ldots ; f_{0}^{\prime}, f_{0}^{\prime \prime}, f_{-1}, \ldots\right)\left(Q_{-1}\right)^{a}|h, q\rangle
$$

is clearly seen to be

$$
\left\langle h_{2}, q_{2}\left|\phi_{h_{3}, q_{3}}(1)\right| \psi\right\rangle=\sum_{a=0} p_{a}\left(h_{2}, h_{3}, 0 \ldots ; q_{2}, q_{3}, 0 \ldots\right) C_{a}
$$

where the constants $C_{a}$ given by

$$
C_{a}=\left\langle h_{2}, q_{2}\left|\phi_{h_{3}, q_{3}}(1)\left(Q_{-1}\right)^{a}\right| h, q\right\rangle
$$

are a priori independent coefficients. Thus there are a countable number of fusions of generic $W_{3}$ representations, with parameters $\left\{C_{a}, a \geq 0\right\}^{2}$. We now consider the case $M_{h_{1} . q_{1} . c}$ reducible.

\subsection{3 -POINT FUNCTIONS AND NULL VECTORS}

As mentioned above the structure of Verma modules over $W_{3}$ is not fully understood yet, as indeed is the case even for simple Lie algebras ${ }^{3}$. There are problems arising from the fact that $Q_{0}$ need not be diagonaliseable (see ref. [11]), and the usual problem that a submodule of a highest weight representation need not necessarily be a sum of highest weight representations (see e.g. ref. [15]). It is conjectured by Bouwknegt et al. that these problems are not present in the representations of the $W_{3}$ algebra which occur in the unitary minimal models which have values of $c$ satisfying

$$
c=2\left(1-\frac{12}{m(m+1)}\right),
$$

where $m$ is an integer greater than 3 (see refs. [16] for details).

\footnotetext{
${ }^{2}$ This is analogous to the situation in the $N=1$ superconformal algebra where in the NeveuSchwarz sector the three point functions $\left\langle h_{2}\left|\phi_{h_{2}}(1)\left(G_{-1 / 2}\right)^{a}\right| h_{3}\right\rangle$ are independent for $a=0,1$ leading to a division of the fusion rules into 'even' and 'odd'

${ }^{3} \mathrm{I}$ would like to thank K. de Vos for pointing this out to me
} 
We shall initially be interested in the cases where $c$ is given by eqn. (2.26) but with $a^{2}$ irrational, and in which there are zero, one, or two independent null vectors in the Verma module $M_{h_{1}, q_{2}, c}$, as in cases (I), (II) and (III) below, although these not an exhaustive list of the possibilities. The diagrams (I-III) show embeddings of Verma modules with the labels being the $L_{0}$ eigenvalues (see e.g. ref. [11] for details). We call representations with one, two and three independent null vectors singly, doubly and completely degenerate respectively.

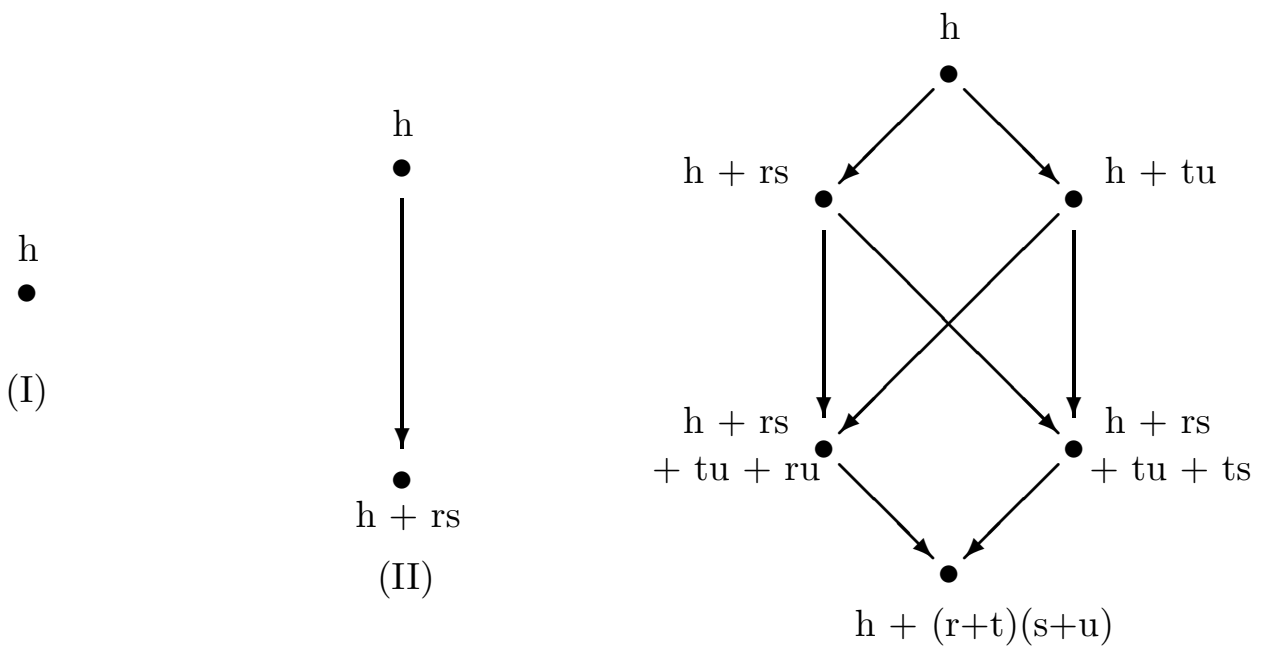

(III)

Case (I). There are no highest weight vectors in $M_{h_{1}, q_{1}, c}$; this case was dealt with in the previous section. There are a countable number of fusions for each set of $\left\{h_{2}, q_{2}, h_{3}, q_{3}\right\}$

Case (II). There is one null vector at level $r s$ in $M_{h_{1}, q_{1}, c}$. If this is of the form ${ }^{4}$

$$
|\psi\rangle=\sum_{a=0}^{N} p_{a}\left(e_{0}^{\prime}, e_{0}^{\prime \prime}, e_{-1}, \ldots ; f_{0}^{\prime}, f_{0}^{\prime \prime}, f_{-1}, \ldots\right)\left(Q_{-1}\right)^{a}\left|h_{1}, q_{1}\right\rangle
$$

with $p_{N}=1$, then requiring this null vector to decouple we find using eq. (2.34),

$$
C_{N}+\sum_{a=0}^{N-1} p_{a}\left(h_{2}, h_{3}, 0 \ldots ; q_{2}, q_{3}, 0 \ldots\right) C_{a}=0
$$

and so $C_{N}$ is given in terms of $C_{a}, 0 \leq a<N$. Similarly by acting on the state (2.37) with $Q_{-1}$ we find that all higher $C_{a}$ are also determined and so there is an $N$-dimensional space of fusions parameterised by $\left\{C_{0}, \ldots, C_{N-1}\right\}$ and no restrictions on the allowed values of $h_{2}, q_{2}, h_{3}, q_{3}$.

Case (III). If there are two independent null vectors these may give rise to a hexagonal pattern of embeddings, and then the situation is more complicated. The presence of two independent null vectors in this way leads to a restriction of the values of $h_{1}, h_{2}, q_{1}$ and $q_{2}$ to a twodimensional curve; that is if $h_{2}$ and $q_{2}$ are given then there is a finite set of allowed values for $h_{3}$ and $q_{3}$.

\footnotetext{
${ }^{4}$ This is certainly the case if one of $r$ or $s$ is 1 , as explicit expressions for the null vectors have been given in $[17]$
} 
The presence of a third independent null vector in the $W_{3}$ minimal models leads to a truncation of the fusions in case (III) to a finite set of allowed values of $\left\{h_{2}, q_{2}, h_{3}, q_{3}\right\}$ in the same way that the presence of a second independent null vector does in the Virasoro minimal models.

\subsection{4-POINT FUNCTIONS}

As before, we can consider a 4-point functions as a sum over chiral blocks, where the intermediate states run over the allowed $W_{3}$ algebra representations in the theory,

$$
B\left(\left(h_{2}, q_{2}\right),\left(h_{3}, q_{3}\right),\left(h_{4}, q_{4}\right),\left(h_{1}, q_{1}\right) ;\left(h_{5}, q_{5}\right)\right)=\sum_{L_{h_{5}, q_{5}}}\left\langle h_{2}, q_{2}\left|\phi_{h_{3}, q_{3}}(1)\right| \psi_{\iota}\right\rangle\left\langle\psi_{\iota}\left|\phi_{h_{4}, q_{4}}(z)\right| h_{1}, q_{1}\right\rangle
$$

If none of the $M_{h_{i}, q_{i}, c}$ are reducible than this chiral block has no restriction on $h_{5}$ and $q_{5}$ and depends on the free parameters $\left\{C_{a}, \tilde{C}_{a}, a \geq 0\right\}$ where

$$
C_{a}=\left\langle h_{2}, q_{2}\left|\phi_{h_{3}, q_{3}}(1)\left(Q_{-1}\right)^{a}\right| h_{5}, q_{5}\right\rangle, \quad \tilde{C}_{a}=\left\langle h_{5}, q_{5}\left|\left(Q_{1}\right)^{a} \phi_{h_{4}, q_{4}}(1)\right| h_{1}, q_{1}\right\rangle .
$$

If $M_{h_{1}, q_{1}, c}$ is reducible with a single null vector, then again the chiral block 2.39 has free parameters $\left\{C_{a}, a \geq 0 ; \tilde{C}_{0} \ldots \tilde{C}_{N-1}\right\}$ and there will be no restriction on $\left(h_{5}, q_{5}\right)$.

If $M_{h_{1}, q_{1}, c}$ has two independent null vectors as in case (III), then this will determine $\tilde{C}_{a}$ up to an overall scale and given $h_{4}, q_{4}$ will restrict $\left(h_{5}, q_{5}\right)$ to lie in a finite set, but the three point function

$$
\left\langle h_{2}, q_{2}\left|\phi_{h_{3}, q_{3}}(1)\right| \psi\right\rangle,
$$

will still depend on the infinite number of arbitrary constants $C_{a}$. So we see that even if one of the fields in a four point function is doubly degenerate, then the 4 point function still is not restricted to lie in a finite space and so will not satisfy a differential equation.

One way we can restrict the 4-point function further is if another of the Verma modules, $M_{h_{2}, q_{2}, c}$ say, is doubly degenerate, as this will again not only force the values of $\left(h_{5}, q_{5}\right)$ to lie in a finite set, but will determine the 3-point functions (2.41) up to an overall constant. It is only then that there will be a finite space of chiral blocks, and that the 4-point functions can satisfy a differential equation in $z$. This is consistent with the results of Bajnok et al. [1] in which differential equations were found for some four point functions of two doubly degenerate fields and two arbitrary fields in the $W A_{2} \equiv W_{3} \equiv W(2,3)$ and $W B C_{2} \equiv W(2,4)$ theories.

Another way to restrict the 4-point function is if $L_{h_{1}, q_{1}, c}$ corresponds to a completely degenerate representation in a $W_{3}$ minimal model. In this case $\left\{h_{2}, q_{2}, h_{5}, q_{5}\right\}$ will be restricted to a finite set of allowed values, each of which corresponds to another allowed minimal model representation. Since $L_{h_{5}, q_{5}, c}$ is now restricted to a minimal model representation, this in turn will restrict $\left\{h_{3}, q_{3}, h_{4}, q_{4}\right\}$ to a finite set of minimal model values.

This clearly generalises to $n$-point functions. If $c$ is not a minimal value, then there will be a finite set of chiral blocks if $n-2$ of the fields are doubly degenerate. If $c$ is a minimal value and one of the fields is in a completely degenerate representation then there will only be a finite set of allowed values of $h_{i}, q_{i}, 1<i \leq n$ and a finite space of chiral blocks for each set of these values. The fusion rules of the minimal $W_{3}$ representations have been given by Frenkel et al. in ref. [18] using the Verlinde formula. 


\section{Conclusion}

We have seen that the structure of correlation functions in theories with $W$-algebra symmetry is rather more complicated than that in a theory with pure conformal symmetry. In particular

the constraints arising from null vectors are weaker, and to obtain differential equations for 4-point functions it is necessary either that at least two of the fields are doubly degenerate, or that one of the representations is completely degenerate, a contrast with the case in Virasoro theory.

I would like to thank K. de Vos, M. Dörrzapf, M. Gaberdiel, A. Kent and F. Malikov for stimulating conversations. I would finally like to thank the organisers for the great efforts they have expended to make this meeting successful and allowing me the chance to present these results.

GMTW was supported by a research fellowship from St. John's College, Cambridge. PB was supported by DOE of USA grant Number DEFG02-90-ER-40560 and NSF grant PHY900036.

\section{References}

[1] Z. Bajnok, L. Palla and G. Takács, A(2) Toda theory in reduced WZNW framework and the representations of the $W$ algebra, Nucl. Phys. B385 (1992) 329; Z. Bajnok, $C_{2}$ Toda theory in reduced WZNW framework, Inst. Theor. Phys. Univ. Budapest preprint ITP-Budapest-501 (1993).

[2] V. G. Kac, Contravariant form for infinite-dimensional Lie algebras and superalgebras, in: Proc. Int. Congress Math. 1978, Helsinki.

[3] A. A. Belavin, A. M. Polyakov and A. B. Zamolodchikov, Nucl. Phys. B241 (1984) 333.

[4] B. L. Feigin and D. B. Fuchs, Representations of the Virasoro algebra, Repts. Dept. Math. Stockholm Univ. (1986), published in 'Representations of infinite-dimensional Lie groups and Lie algebras', eds. A. Vershik and D. Zhelobenko, Gordon and Breach (1989).

[5] B. L. Feigin and D. B. Fuchs, Functional Analysis and its Applications 16 (1982) 114.

[6] B. L. Feigin and D. B. Fuchs, J. Geom. Phys. 5 (1988) 209.

[7] R. P. Langlands, Commun. Math. Phys. 124 (1989) 261.

[8] M. Bauer, P. di Francesco, C. Itzykson and J.-B. Zuber, Phys. Lett. B260 (1991) 323. Nucl. Phys. B362 (1991) 515;.

[9] A. Kent, Phys. Lett. 278B (1992) 443.

[10] A. B. Zamolodchikov, Theor. Math. Phys. 65 (1985) 347.

[11] G. M. T. Watts, Determinant formulae for extended algebras in two-dimensional conformal field theory, Nucl. Phys. B326 (1989) 648; erratum Nucl. Phys. B336 (1989) 720 .

[12] V. A. Fateev and A. B. Zamolodchikov, Nucl. Phys. B280 [FS18] (1987) 644. 
[13] V. A. Fateev and S. L. Luk'yanov, Int. J. Mod. Phys. A3 (1988) 507; Sov. Sci. Rev. A15 (1990) 1.

[14] F. A. Bais, P. Bouwknegt, K. Schoutens and M. Surridge, Nucl. Phys. B304 (1988) 348; Nucl. Phys. B304 (1988) 371.

[15] I. N. Bernstein, I. M. Gelfand and S. I. Gelfand, Differential operators on the base affine space and a study of $g$-modules, in: Proc. 1971 Summer School in Math., Janos Bolyai Math. Soc. Budapest, ed. I. M. Gelfand, pages $21-64$.

[16] P. Bouwknegt, J. McCarthy and K. Pilch, Semi-infinite cohomology of W-algebras, University of Southern California preprint USC-93/11 (1993); On the BRST structure of $W_{3}$ gravity coupled to $c=2$ matter, University of Southern California preprint USC93/14 (1993), To appear in Proc. of AMS Special Session on Geometry and Physics, Los Angeles, Nov 1992.

[17] P. Bowcock and G. M. T. Watts, Phys. Lett. 297B (1992) 282.

[18] E. V. Frenkel, V. Kac and M. Wakimoto, Characters and fusion rules for W-algebras via quantised Drinfel'd-Sokolov reductions, Commun. Math. Phys. 147 (1992) 195. 